\title{
Mesenchymal stem cells: from stem cells to sarcomas
}

\begin{abstract}
Mesenchymal stem cells (MSCs) have garnered vast interests in clinical settings, especially in regenerative medicine due to their unique properties $ठ$ they are reliably isolated and expanded from various tissue sources; they are able to differentiate into mesodermal tissues such as bones, cartilages, adipose tissues, and muscles; and they have unique immunosuppressive properties. However, there are some concerns pertaining to the role of MSCs in the human body. On one hand, they are crucial component in the regeneration and repair of the human body. On the contrary, they are shown to transform into sarcomas. Although the exact mechanisms are still unknown, many new leads have pointed to the belief that MSCs do play a role in sarcomagenesis. This review focuses on the current updates and findings of the role of MSCs in their transformation process into sarcomas.
\end{abstract}

Keyword: Mesenchymal stem cell; Oncogene; Regenerative medicine; Sarcoma; Spontaneous transformation 